\title{
LOS BRIÓFITOS DEL HERBARIO DE WILLKOMM (COI). II. MUSCI
}

\author{
$\mathrm{M}^{\mathrm{a}}$ Carmen VIERA y Juan REINOSO
}

\begin{abstract}
RESUMEN. Briófitos del Herbario de Willkomm (COI). II. Musci. En este trabajo se estudia la colección de musgos del herbario de Willkomm (COI). Se trata de 90 taxones que se encuentran en la carpeta $\mathrm{n}^{\circ} 4$ de dicho herbario (Musci Frondosi). No se tiene noticia de que la colección hubiese sido señalada previamente en la bibliografía briológica, se considera por lo tanto inédita.
\end{abstract}

Palabras clave. Herbario, Willkomm, Briófitos, Musgos.

RESUMÉ. Bryophytes de l'Hervier Willkomm (COI). II. Musci. Dans ce travail on étudie la collection des mousses présent dans l'herbier Willkomm (COI). Il s'agit de 90 taxons qui se trouvent dans la carpette $\mathrm{n}^{\circ}$ 4 (Musci Frondosi). La collection n'avait pas êtê cité auparavant dans la bibliographie bryologique, on considére pourtant inédite.

Mots clés. Herbier, Willkomm, Bryophytes, Mousses.

\section{INTRODUCCIÓN}

Con este trabajo continuamos el estudio de la revisión de los briófitos del herbario de Willkomm ya iniciado anteriormente (Viera y Reinoso, 1993).

Para ello, durante los años 1990 y 1991 en diferentes estancias en el «Instituto Botânico Dr. Julio Henriques da Universidade de Coimbra (Portugal)», hemos llevado a cabo la revisión de los musgos de dicho herbario. El material objeto de estudio está contenido en la carpeta número 4 de la colección (Musci Frondosi). Después de efectuada la revisión el total de taxa contabilizados asciende a 90 .

$\mathrm{Al}$ igual que en el caso de las hepáticas, buena parte de dicho material fue recolectado por Willkomm en sus campañas llevadas a cabo por diferentes provincias españolas como Valencia (Cerro de Santa María en la Sierra de Chiva, Albufera, etc), Granada (La Alhambra, el Generalife y Sierra Nevada : barranco de Trévelez, barranco de San Juan, borreguil de Dilas, corral del Veleta, etc) entre otras Willkomm (1844, 1851 y 1852), Kunze (1846).

Además del material recogido por Willkomm merece destacar en primer lugar, las aportaciones a dicho herbario del botánico

Realizado en el marco del Proyecto Nr. 3747/91 subvencionado por el Vicerrectorado de Investigación de la Universidad de Santiago de Compostela. 
aragonés F. Loscos.

Son muy numerosos los ejemplares de musgos que Loscos envía a Willkomm, no sólo como intercambio de duplicados sino también, como ocurre en la mayor parte de los casos, para resolver problemas taxonómicos. Con frecuencia Loscos escribe en la etiqueta del ejemplar junto con el nombre de la especie, comentarios de interés diagnóstico y descripciones detalladas, tal es el caso de Aloina rigida y Grimmia orbicularis, entre otras.

Es interesante señalar que en la colección de briófitos del herbario de Willkomm existe una buena representación de musgos macaronésicos procedentes básicamente de las colecciones de Mandon: "Mousses de Madère» (Ribero Frio, Sideras, Picos las Arrieras, Ribera de Metade, etc) y de Bourgeau: » Pl. Canarienses» (Tenerife: Font del Agua García, Los Organos, Guimar, etc).

Finalmente dicha colección de musgos contiene también una representación interesante de especies africanas y europeas, procedentes de colecciones de otros herbarios: F. Schultz (Herbarium Normale), Argelia; Soleirol (Herb. Cors.); Orphanides (Flora Graeca); Lenormand (Flora Galliae et Germaniae) y Dr. C. Baenitz (Herbarium Europaeum) con las aportaciones de los recolectores siguientes: Heldreich (Herbar. Florae Hellenicae y Flora Attica), Arcangeli, Marcucci y Levier.

Al igual que comentábamos en nuestro trabajo anterior, destacan las recolecciones de Willkomm por el buen estado de conservación en que se encuentran, llevando anotaciones manuscritas del mismo autor en los sobres. Por el contrario, también queremos señalar las no muy buenas condiciones de una parte del material restante, muy desmenuzado y descuidado su etiquetado. De manera que hemos procedido a su debida restauración manteniendo escrupulosamente todas las anotaciones y etiquetas de los diferentes autores y recolectores.

Para la identificación de los taxones, no- menclatura y ordenación en el catálogo de los mismos, se han seguido los trabajos de Bruch, Schimper \& Gumbel (1836-51); Casares Gil (1932); Casas (1981); Corley, Crundwell, Düll, et al. (1981); Crum \& Anderson (1981); Demaret \& Castagne (1968); Düll (1980); Husnot (1884-90); Limpricht (1895); Monkemeyer (1927); Paris (1904); Smith (1980) y Wijk, Margadant \& Florschutz (1959. 69), entre otros.

Para cada taxón se indica el nombre correcto, sinónimo de interés, nombre con el que aparece en el pliego y etiqueta(s) que acompaña al(los) ejemplar(res).

Por último queremos resaltar que para la transcripción de las etiquetas hemos seguido las normas habituales de transcripción (Candollea, 36: 543-548, 1981).

\section{MUSGOS DEL HERBARIO DE WILLKOMM}

1) Sphagnum capillifolium (Ehrh.)Hedw., Fund. Musc. 2: 86 (1782). (= Sphagnum palustre var. capillifolium Ehrh., Hannover Mag. 15: 235(1780)).

a) S. acutifolium Ehrh.

Etiqueta: [m. Willkomm]: Sphagnum acutifolium Ehrh. / Hab. in Aragonia prope Lumbreras. / Ex herb. d. Boutelou 1844. I

2) Sphagnum compactum Lam. \& DC., Fl. Franc., ed. 2,2: 443 (1805)

a) Sphagnum compactum

Etiqueta: [impreso]: soleirol, herb. cors. // [m. i. ]: 5039. Sphagnum compactum / Mont. Coccione. 1

3) Pogonatum nanum (Hedw.) P. Beauv., Prodr. 84 (1805). (=Polytrichum nanum Hedw., Spec. Musc. 95 (1801)).

a) Polytrichum nanum $\mathrm{DC}$.

Etiqueta. - [impreso]: soleirol, herb. cors. // [m. i. ]: 5014. Polytrichum nanum DC. / Galeria / b) Polytrichum nanum Schreb.

Etiqueta. - [impreso]: E. Bourgeau. Pl. Canarienses 1855. // Polytrichum nanum Schreb. Phyt. / Con. IV, 26. / (Mont.) / Teneriffa, bordes du 
chemín a Cigueste / 7 Fevrier. /

4) Pogonatum aloides (Hedw.) P. Beauv., Prodr. 84 (1805). (= Polytrichum aloides Hedw., Spec. Musc. 96 (1801)).

a) Pogonatum aloides Brid.

Etiqueta. - [m. Mandon]: Mandon: Mousses de Madère // Pogonatum aloides Brid. / Ribero Frio. 1 Mai. $n^{\circ} 27.1$

b)Polytrichum aloides DC.

Etiqueta. - [impreso]: soleirol, herb. cors. // [m. i. ]: 5016 a. Polytrichum aloides DC. / Calvi Septembre. 1

5) Polytrichum formosum Hedw., Spec. Musc. 92.

19 f. 1a (1801)

a) Polytrichum formosum Hopp.

Etiqueta 1. - [impreso]: Herbarium Willkomm. / [m. Willkomm]: Polytrichum formosum Hopp. / [impreso]: Legi 18 [m. Willkomm]: 50

Etiqueta 2. - [m. Willkomm]: Cr. A. / Polytrichum / formosum Hoppe /

6) Polytrichum juniperinum Hedw., Spec. Musc. 89. 18 f. 6-10. (1801)

a) Polytrichum juniperinum Hedw.

Etiqueta 1. - [m. Willkomm]: Polytrichum juniperinum Hedw. / Hab. in Sierra Nevada (tachado): in ad (sin tachar): in / regione alpina: ad rivulos partis su- / perioris Dehesae de S. Gerónimo / promirone cum Bryum pallescenti / ad alt. circ. 7000'. / legi d. 31 Julii 1844. I

Etiqueta 2. - [m. Willkomm]: Polytrichum juniperinum Hedw. / Hab. in Sierra Nevada in pratis / alpinis vallis barranco de Trevelez, / ad alt. circ. 7000'. / Legi d. 17 Sept. 1844. I

Etiqueta 3. - [manuscrito]: E. Bourgeau. Pl. Canarienses 1855. // Polytrichum juniperinum Willd. / (Mont.) / Teneriffa a Cegueste 28 Janvier./

Etiqueta 4. - [m. Mandon]: Mandon: Mousses de Madère. // Polytrichum juniperinum Hedw. / Ribero Frio. avril. n 29.

7) Fissidens incurvus Starke ex Röhl., Deutsch. Fl. Krypt. Gew - ed. 2, 3: 76(1813)

Etiqueta. - [manuscrito]: Fissidens incurvus / [m. Willkomm]: Aragonia austr. / Legi Loscos / Schutz I

8) Fissidens taxifolius Hedw., Spec. Musc. 155. 39f. 1-5 (1801)

Etiqueta. - [manuscrito]: E. Bourgeau. Pl. Canarienses 1855. // Fissidens taxifolius Hedw. / (Mont.) / Teneriffa, font del Agua Garcia / 4 mars./

9) Fissidens adianthoides Hedw., Spec. Musc. 157 (1801)

Etiqueta 1. - [impreso]: Herbarium Willkomm. / [m. Willkomm]: Fissidens adiantoides Hedw. / Aragon. australis./ [impreso]: Legi [m. Willkomm]: t Loscos [impreso]: $18 /$

Etiqueta 2. - [manuscrito]: $339 /$ [m. Willkomm]: Fissidens / adiantoides Hedw. /

10) Fissidens serrulatus Brid., Spec. Musc. 1: 170 (1806)

Etiqueta 1. - [impreso]: E. Bourgeau, PL. CANARIENSES (ex itinere secundo) 1855 // 1583. FISSIDENS SERRULATUS Brid. - Phyt. Can. IV, 22. / (Montagne.) / Teneriffa: Selva del Agua Garcia, ad rivulos. 4 martii. $/$

Etiqueta 2. - [manuscrito]: Mandon: Mousses de Madère. //Fissidens serrulatus Brid. / Ribero Frio 6 fevrier. $\mathrm{n}^{\circ} 5.1$

11) Dicranum scoparium Hedw., Spec. Musc. 126 (1801)

Etiqueta 1 - [impreso]: Herbarium Willkomm. / [m. Willkomm]: Dicranum scoparium Ligos. / [impreso]: Legis 18 [m. Willkomm]: $50 . /$

Etiqueta 2. -[m. Willkomm]: Dicranum / 19./ scoparium Legis /

12) Dicranum scottianum Turn., Musc. Hib. 75.6

f. 1. (1804)

Etiqueta 1. - [impreso]: E. Bourgeau, PL. CANARIENSES (ex itinere secundo), 1855.// 1589. DICRANUM SCHOTTIANUM Turner. - Phyt. Can. IV, 38. / (Montagne.) / Teneriffa: Selva del Agua Garcia, in petrosis. 4 martii. $/$

Etiqueta 2. - [manuscrito]: Mandon: Mousses de Madère. // Dicranum Scottianum Turn. / Sideras 29 Junè. $n^{0} 3.1$

13) Oncophorus virens (Hedw.) Brid., Bryol. Univ. 1: 399 (1826). (= Dicranum virens Hedw., Spec. Musc. 142 (1801)).

Etiqueta 1. - [impreso]: Herbarium Willkomm. / [m. Willkomm]: Dicranum virens / Aragonia australis. / [impreso]: Legi [m. Willkomm]: t Loscos 
[impreso]: $18 /$

Etiqueta 2. - [m. i. ]: $332 /$ [m. Willkomm]: Dicranum / virens Hedw. ? /

Etiqueta 3. - [m. Willkomm]: Dicranum virens Hedw. / Flor. Hispan. / In ? Sierra nevada an ? Rollan / du fisurangius: Corral de Veleta, Val / de Casillas. 8 -1000'. / Jul. Aug. 1844. /

Etiqueta 4. - [m. Willkomm]: Dicranum virens Hedw. / Hab. in Sierra Nevada / in valle barranco de Val de Casillas / ad alt. 8000'. / Legi d. 23 Aug. 1844. I

Etiqueta 5. - [m. Willkomm]: Dicranum virens Hedw. / Crypt. hisp. exsicc. n. 20. (935). / Hab. in Sierra Nevada locis humidis umbrosis regionis nivalis: / in Corral de Veleta ad ruper madidas inter glacier. / 9000- 9500'. / Legi d. 26 Julii 1844. I

14) Ceratodon purpureus (Hedw.)Brid., Bryol. Univ. 1: 480(1826). (= Dicranum purpureum Hedw., Spec. Musc., 136, tab. XXXVI. (1801)).

a) Ceratodon purpureus Brid.

Etiqueta. - [manuscrito]: E. Bourgeau. Pl. Canarienses 1855 // Ceratodon purpureus Brid. / (Mont.) / Teneriffa, foset de las Mercedès / 10 février. I

15) Distichium capillaceum (Hedw.) B. S. G., Bryol. Eur. 2 (29-30): 156. 193. (1846). (= Cynodontium capillaceum Hedw., Spec. Musc. 57 (1801)).

a) Distichium capillaceum Br. et Sch.

Etiqueta . - [m. Willkomm]: Distichium capillaceum Br. et Sch. / Hab. in Sierra Nevada locis umbrosis / humidis regionis nivalis: in Corral de / Veleta in consortio Dicrani virensis. / 9000 9500'. / Legi de. 26 Julii 1844. /

16) Encalypta rhaptocarpa Schwaegr., Spec. Musc. Suppl. 1(1): 56. 16(1811)

a) E. spathulata C. Müll.

Etiqueta . [m. Willkomm]: Encalypta spathulata C. Müll. / (tachado): vulgaris Hedw. / pl. rarissima ! Crypt. hisp. exsicc. 14. (931). / Hab. in fissuris rupium calcarearum umbrosis / cacuminis Cerro de Sta. Maria in Sierra de Chiva regni Valentini. 5500 - 6000'. / Legi d. 2 Junii 1844. /

17) Tortula laevipila var. meridionalis (Schimp.)Wijk \& Marg., Taxon 8: 75(1959). (= Tortula laevipila (Brid.)Schwaegr., Spec. Musc.
Suppl. 2(1): 66 (1823) subsp. laevipilaeformis (De Not.) Giac., Atti Inst. Bot. Univ. Lab. Critt. Pavia ser. 5, 4: 216(1947)).

a) Tortula laevipila Hedw.

Etiqueta. - [manuscrito]: E. Bourgeau. P1. Canarienses 1855. // Tortula laevipila Hedw. / (Mont.) / Teneriffa, aus Organos 16 mars. /

18) Tortula subulata Hedw., Spec. Musc. 122. $27 \mathrm{f}$. 1-3. (1801).

Barbula subulata Brid.

Etiqueta. -[m. Willkomm]: Barbula subulata Brid. / Crypt. hisp. exsicc. n. 44. (938). / Hab. in Sierra Nevada in rupibus / schistosis madidis partis superioris / vallis barranco de S. Juan, ad alt. $/ 7000$ - 7500'. / legi d. 3 Sept. 1844

19) Tortula inermis (Brid.) Mont., Arch. Bot. 1: 136, 4f. 4 (1832). (= Syntrichia subulata var. inermis Brid., Bryol. Univ. 581 (1826)).

a) Barbula inermis

Etiqueta 1. - [impreso]: Herbarium Willkomm. / [m. Willkomm]: Barbula inermis / Aragonia australis / [impreso]: Legit [m. Willkomm]: Loscos [impreso]: 18

b) Barbula (Tortula) inermis

Etiqueta 2. - [m. Willkomm]: Barbula (Tortula) inermis / mit pusilum Didymodon lividus /

c) Tortula uninervifolia

Etiqueta 3. - [m. Willkomm, tachado]: Tortula / uninervifolia / [m. Willkomm]: Barbula / inermis Mont.

20) Tortula cuneifolia (With.) Turn., Musc, Hib. 51 (1804) (= Bryum cuneifolium With., Syst. Arr. Brit. Pl. ed. 4, 3: 794 (1804)).

a) Tortula cuneifolia Hook.

Etiqueta. -[manuscrito]: E. Bourgeau. Pl. Canarienses 1855. // /Tortula cuneifolia Hook. et Tayl. / (Mont.)/ Teneriffa: Guimar / 18 Fèvrier. /

21) Tortula muralis Hedw., Spec. Musc. 123(1801)

a) Barbula muralis Timm.

Etiqueta 1. - [m. Willkomm]: Barbula muralis Timm. / Crypt. Hisp. exsicc. n. 88 (939). / Hab. in muris arenosisque isthmi gaditani copiose / Legi d. 27 Januarii 1845 /

b) Syntrichia muralis Brid.

Etiqueta 1. - [m. Willkomm]: 23. / Syntrichia muralis / Brid. / 
Etiqueta 2. - [impreso]: Herbarium Willkomm. / [m. Willkomm]: Syntrichia muralis Brid. / [impreso]: Legi 18 [m. Willkomm]: $50 /$

22) Tortula atrovirens (Sm.) Lindb., Oefv. K. Vet. Ak. Foerh. 21(4) : 236 (1864). (= Grimmia atrovirens Sm., Engl. Bot. 28: 2015 (1809)).

a) Trichostomum convolutum

Etiqueta 1. - [m. Willkomm] : Trichostomum convolutum / [m. Loscos]: Prusia // Sr. D. Mauricio Willkomm / Tharant / (cerca de Dresde) /

Etiqueta2. -[m. Loscos]: Gen. Willkommia / con cerda terminal / caperuza gemadas / Peristoma sencillo con / 32 dientes, filiformes equi / distantes, libres por la base. / - / Tallo sencillo muy corto / Hojas abiertas, elipticas o no / nervadas apiculadas poe el/ nervio principal. Opercu / lo corto, conico?/

Etiqueta 3. - [m. Loscos]: Willkommia / [m. i. ]: Trichostomum convolutum /

b) Desmatodon nervosus Bruch et Sch.

Etiqueta . - [impreso]: F. Schultz, herbarium normale. Cent. 2. // 196, DESMATODON NERVOSUS (Didymodon Hook et / Toyl. musc. brit. 115, t. 20. Brid. br. un. I. / 516) Bruch et Sch. br. eur. I. I. t. III., R. D. / K. Fl. 2. p. 100. / Mars 1851. / Sur les murs d'ardoise servant de clôture aux environs / d'Angers (Maine et Loire). / Rec. Guépin. I

23) Aloina aloides var. ambigua (B. S. G.) Craig in Grout, Moss. Fl. N. Am. 1(4): 214 (1939). (= Barbula ambigua B. S. G., Bryol. Eur. 2: 76. 139 (1842)).

a) Barbula ambigua Br. \&Schimp.

Etiqueta. - [m. Heldreich): Dr. C. Buenitz, Herbarium Europaeum. / De Heldreich, Herbar. Florae Hellenicae $N^{\circ}$ 98. / Barbula ambigua Br. \& Schimp. / (Fide Geheeb!) / Flora Attica: in maceriis pr. / Expolia Oliveti Athenarum / legit th. de Heldreich. / d. 11 Dec. 1876

24) Aloina rigida (Hedw.) Limpr., Laubm. Deutschl. 1: 637 (1888). (= Barbula rigida Hedw., Spec. Musc. 115 (1801)).

a) Tortula rigida

Etiqueta 1. - [m. Loscos]: Tort. rigida /

Etiqueta 2. - [m. Loscos]: Tortula rigida / Especie vulgar: las hojas de esta / planta se confunden facilmente / con las del Didymodon luridum /? el fruto Marzo / Hab. en toda la tierra baja / tambien en Peñarroya /

Etiqueta 3. - [m. Loscos]: 70/Series inconfecta / es o no la T. rigida? /

Etiqueta 4. - [m. Loscos]: Tortula / (tachada): rigida [m. i. ]: aloides /

b) Barbula rigida Schultz.

Etiqueta. - [m. Willkomm]: Barbula rigida Schultz. / Crypt. hisp. exsicc. $n^{\circ}$ 6. (941). / Hab. in regno valentino in lutosis / umbrosis humidis in ripa juxta / vallerulae barranco de Tabernas. / legi d. 16 Maji 1844.

25) Pterygoneurum ovatum (Hedw.) Dix., Rev. Bryol. Lichénol. 6: 96 (1934). (= Gymnostomum ovatum Hedw., Spec. Musc. 31. 2 f 1-3 (1801)).

a) Gymnostomum ovatum; Pottia cavifolia

Etiqueta . -[m. Loscos, tachado]: Gymnosth. / ovatum [m. Willkomm]: Chorda / [m. i. ]: Pottia cavifolia /

26) Crossidium squamiferum (Viv.) Jur., Laubmfl. Oest. Ungarn

(= Barbula squamigera Viv., Annali Bot. 1(2): 191 (1804)).

a) Tortula membranifolia Hook.

Etiqueta 1. -[m. Loscos]: Tortula / (tachado): chloronotos / [m. i. ]: membranifolia / Hook /

Etiqueta 2. - [m. Loscos]: Tortula (tachado): chlorono / tos Brid. / [m. i. ]: membranifolia /

Etiqueta 3. - [impreso]: Herbarium Willkomm. / [m. Willkomm]: Tortula membranifolia Hook. / Aragonia australis. / [impreso]: Legi [m. Willkomm]: t Loscos [impreso]: 18

Etiqueta 4. - [m. Loscos]: Tortula (tachado): chloro / notos Brid. / invierno / [m. i. ]: membranifolia /

27) Pottia lanceolata (Hedw.) C. Müll., Syn. 1: 548 (1849)

(= Encalypta lanceolata Hedw., Spec. Musc. 63 (1801)).

a) Pottia lanceolata; Didymodon apiculatum

Etiqueta 1. - [m. Loscos]: 64 / [m. i. ]: Pottia lanceolata / [m. Loscos]: veanse las Weissia colocadas en hb. / dientes 32, reunidos por pares / Didymodon? / los dientes son ademas, unidos en anillo / por la base. / Lo es Didymodon / apiculatum/

Etiqueta 2. - [m. i. ]: Pottia lanceolata / [m. Loscos tachado]: Willkommia / (sin tachar de Loscos): Hojas aovadas concavas / apiculadas, pro- 
vistas de un / grueso y ancho nervio dorsal / 32 pestañas libres / una Tortula? /

Etiqueta 3. - [m. Loscos]: 67 / [m. i. ]: Pottia lanceolata [m. Willkomm]: C. Müll. / [m. Loscos tachado]: Didymodon apiculatum ?

b) Caperma demediata

Etiqueta . - [m. Loscos]: 63 / Caperma demediata $/$

28) Phascum cuspidatum Hedw., Spec. Musc. 22 (1801)

a) Phascum cuspidatum Schreb.

Etiqueta 1. - [m. Loscos]: Phascum cuspidatum / Schreb. 12 abril 1869 / Herb. de Loscos /

Etiqueta 2. - [m. Loscos ]: Phascum cuspidatum / Schreb. 18 abril 1869 / Herb. de Loscos $/$

Etiqueta 3. - [m. Loscos]: Phascum cuspidatum. / [m. i. ]: ?

29) Phascum cuspidatum var. piliferum (Hedw.)Hook. \& Tayl., Musc. Brit. 8(1818). (= Phascum piliferum Hedw., Spec. Musc. 20 (1801)).

a) Phascum cuspidatum var. piliferum

Etiqueta. - [m. Loscos]: Planta rarisima $99 /$ [m. i. ]: Phascum cuspidatum ad var. piliferum / attedens / [m. Loscos tachado]: Phascum curvicollum / Hedw. ??. [m. Loscos sin tachar]: Peristoma sencillo / los dientes pocos, fugaces. / con la urna rugosa re / ticulada en la superficie / Marzo, Peñarroya junto / a las paredes del pueblo / Hojas con nervio prolongado más /allá del apice. [m. Willkomm]: Leg. Loscos. /

30) Acaulon triquetrum (Spruc.)C. Müll., Bot. Zeit. 5: 100. 1847). (= Phascum triquetrum Spruce., London J. Bot. 4: 189 (1845)).

a) Phascum triquetrum Spruc.

Etiqueta. - [m. Loscos] / : muticum / [m. i. ]: triquetrum [m. Willkomm]: Spruce. / Aragon. / Leg. Loscos. I

31) Barbula unguiculata Hedw., Spec. Musc. 118 (1801)

a) Tortula unguiculata Hedw.

Etiqueta. - [m. Loscos]: 69 / Tortula unguiculata / Hedw. Abril 1869 / Herb. de Loscos / [m. i. ] ?): ? !/?/.
32) Eucladium verticillatum (Brid.)B. S. G., Bryol. Eur. 1(33- 36): 9. 40(1846). (= Weissia verticillata Brid., J.f. Bot. 1800(2): 283 (1801)).

a) Eucladium verticillatum Br. Eur. var. setaceum Sch.

Etiqueta. - [m. Mandon]: Mandon: Mousses de Maderè // Eucladium verticillatum Br. Eur. / var. setaceum Sch. / Picos las Arrieras. 5 mai. ${ }^{\circ}$ 6. 1

b) Weissia verticillata Schw.

Etiqueta - - [m. Willkomm]: Weissia verticillata Schw. / Crypt. hisp. exsicc. ${ }^{\circ}$ 42. (934) / Hab. in calcaris cavernarum humidarum valleculae interavicum Alhambra et villam / et Ginaraliphe prope arcum aquaeductus copiose, sed semper fluilis / Alt. 2500'. / Legi d. 20 Julii 1844. /

33) Weissia controversa Hedw., Spec. Musc. 67 (1801)

a) Weissia viridula Brid.

Etiqueta . [manuscrito] : E. Bourgeau. P1. Canarienses 1855. // Weissia viridula Brid. / (Mont.) / Teneriffa, foret de las Mercedès / 10 Février. /

34) Weissia wimmeriana (Sendth.) B. S. G., Bryol. Eur. 1(33-36): 64. 20 (1846). (= Gymnostomum wimerianum Sendth., Flora 23: 59 (1840)).

a) Weissia wimmeriana

Etiqueta . - [m. Loscos]: Dicranum / squarrosum ?? / [m. Willkomm]: Aragonia austral / Leg. Loscos / [m. i. ]: Weissia Wimmeriana / gymnostoma /

35) Weissia condensa (Voit) Lindb., Oefv. K. Vet. Ak. Foerh. 21: 230 (1863). (= Gymnostomum condensum Voit in Sturm, Deutschl. Fl. 2(11). ic (1811)).

a) Hymenostomum tortile

Etiqueta 1. - [impreso]: Herbarium Willkomm / [m. Willkomm]: Hymenostomum tortile / Aragonia australis. / [impreso]: Legi [m. Willkomm]: / Loscos [impreso]: $18 /$

Etiqueta 2. - [m. i. ]: 59 / : Gimnostomum rupestre / [m. Willkomm]: Brid. / [m. i. ]: Hymenostomum tortile /

b) Gymnostom rupestre

Etiqueta 1. - [m. Loscos]: 58 / Gymnostomum rupestre / Schw. 31 Marzo, fr. jov. / Herb. de Loscos /

Etiqueta 2. - [m. i. ]: (tachado): Gimnosthomum / rupestre ? / 59 / [m. i. ]: 
Hymenosthomum tortile /

36) Tortella tortuosa (Hedw.) Limpr., Laubm. Deutschl. 1: 604 (1888). (= Tortula tortuosa Hedw., Spec. Musc. 124 (1801)).

a) Barbula tortuosa W. et M. var. brevicaulis Schw.

Etiqueta. - [m. Willkomm]: Barbula tortuosa W. et M. I var. brevicaulis Schw. / Crypt. hisp. exsicc. $n^{\circ}$ 5. (940)/ Hab. in arenosis sub. pinetis inter lacum / Albufera et mar in regno Valentino / legi d. 13 Maji 1844.

37) Timmiella barbuloides (Brid.) Mönk., Laubm. Eur. 273 (1927). (= STrichostomum barbuloides Brid., Spec. Musc. 1: 233 (1806)).

a) Trichostomum barbula Schw.

Etiqueta 1. -[manuscrito]: E. Bourgeau. Pl. Canarienses 1855. // Trichostomum Barbula Schwaegr. / (Mont.) / Teneriffa, aus Organos 12 Mars. I

Etiqueta 2. -[m. Willkomm]: Trichostomum barbula Schw. / Crypt. hisp. exsicc. 103. (937) / Hab. in parte superiore montis / Sierra de Palma in dechinibus / humosis umbrosis humidis / 2 -3000'. / Leg. d. 22 Martii 1845. /

38) Cinclidotus mucronatus (Brid.) Mach., Cat. Descr. Briol. Portug. 57. (1919). (= Dialytrichia mucronata (Brid). Brot. cf. Limpr., Laubm. Deutschl. 1: 691 (1888)).

Barbula mucronata Brid., Sp. M. 1: 268 (1806)

a) B. brevissonii Brid.,

Etiqueta. - [m. i. ]: Dr. C. Baenitz Herbarium Europaeum / No. / Barbula brebissonii Brid., / c. fr. $/ \mathrm{ad}$ pedem arborum annosarum in R. Viridario Boboli Florentiae / Majo 1876 legit J. Arcangeli

39) Cinclidotus riparius (Brid.)Arnott, Mem. Soc. Linn. Paris 7: 247(1827). (=Gymnostomum riparium Brid., J. f. Bot. 1800 (2): 274 1801)).

a) Racomitrium riparium Brid.

Etiqueta . - [m. Willkomm]: Racomitrium riparium Brid. ? / Crypt. hisp. exsicc. n. 36 (946) / Hab. in Sierra Nevada ad rivulos regionis (tachado): alp. (sin tachar): nivalis: / en el borreguil de $S$. Gerónimo, ad alt. 8000 - 8500'. / Legi d. 3 Aug. 1844. I

40) Coscinodon cribosus (Hedw.) Spruce., Ann.
Mag. Nat. Hist. ser. 2, 3: 491(1849). (=Grimmia cribosa Hedw., Descr. III, 73(1): 31 A (1792)).

a) Coscinodon pulvinatus Spreng.

Etiqueta . -[impreso]: F. Schultz, herbarium normale. Cent. 2. // 191, COSCINODON PULVINATUS, Spreng. Anl. III. / 281. t. 8. F. 75, R. D. K. Fl. 2. p. 173; C. / Persoonii, Hampe; Grimmia cribosa (Pers.) / Hedw. musc. frond. III. 73, Brid. br. un. I. / 174, Duby 574. / Mars 1851. / Sur les murs d'ardoise bâtis à froid, près d'Angers (Maine- / et - Loire) / Rec. Guúpin. /

41) Grimmia alpestris (Web. \& Mohr) Schleich. ex Hornsch. in Sommerf., Suppl. Fl. Lapp. 49 (1826). (=Trichostomum pulvinatum var. alpestris Web. \& Moh., Bot. Taschb. 110 (1807)). Etiqueta . - [manuscrito]: Panticosa / 6/50/

42) Grimmia pulvinata (Hedw.) Sm., Engl. Bot. 24: 1728 (1867) IV. (= Fissidens pulvinatus Hedw., Spec. Musc. 158. 40 f. 1-3. (1801)).

a) Grimmia pulvinata

Etiqueta 1. - [m. Loscos]: Grimmia pulvinata / Sm. Marzo, abril 1869 / Herb. de Loscos /

Etiqueta 2. - [m. i. ]: Grimmia / pulvinata / ? 621

Etiqueta 3. - [m. i. ]: Parecesen / Grimmia /

Etiqueta 4. - [m. i. ]: Grimmia / pulvinata / en compañia de la esp. / desconocida /

Etiqueta 5. - [m. i. ]: Grimmia / pulvinata / ?

43) Grimmia orbicularis Bruch ex Wils., Engl. Bot. Suppl. 4: n. 2888. (1844).

a) Grimmia orbicularis

Etiqueta 1. - [m. Loscos]: Desconocida / Grimmia orbicularis / Peristoma sencillo / con 16 dientes, al fin / 32. Cerda terminal / caperuza denudada /esp. vulgarisima/

Etiqueta 2. -[m. Loscos]: $66 /$ desconocido / vulgarisimo: muy semejante a la / Grimmia pulvinata / cerda terminal, caperuza denudada / peristoma sencillo 16 dientes equi / distantes, vease si son enteros o no / esos dientes. I

Etiqueta 3. -[m. Loscos]: Desconocida, comunísima, muy semejante a la grimmia / pulvinata

Etiqueta 4. - [m. Rodriguez]: : 66 / Grimmia / orbicularis / Hampe /

Etiqueta 5. -[m. Rodriguez]: Tal vez Loscos ha confundi / do dos especies congeneres en / una sola. / Este grupo parece tener urnas / mas gruesas, 
y de aspecto dife / rente de los grupos restantes. /

44) Grimmia trichophylla Grev., Fl. Edinensis 235 (1824).

a) Grimmia pulvinata Lind.

Etiqueta . - [manuscrito]: Dr. C. Baenitz, Herbarium Europaeum. // De Heldreich, Herbar. Florae Hellenicae. No 99. // Grimmia pulvinata Lin. (subBryo) / Smith. var. / Flora Attica: in rupibus m. Corydali prope Daphne. D. 18 Dec. 1876/ Legit Th. de Heldreich. 1

45) Racomitrium heterostichum (Hedw.) Brid., Mant. Musc. 79 1819). (=Trichostomum heterostichum Hedw., Spec. Musc. 129 (1801)).

a) Trichostomum heterostichum Hedw.

manuscrita de Willkomm): Trichostomum heterostichum Hedw. / (letra impresa): Legi 18 (letra manuscrita de Willkomm): 50 /

Etiqueta 2. - [m. Willkomm]: Trichostomum / heterostichum Hedw. / 20. I

Etiqueta 3. - [impreso]: Herbarium Willkomm. / [m. Willkomm] : Trichostomum heterostichum Hedw. / Aragonia australis / [impreso] : Legi [m. Willkomm] : t Loscos. (letra impresa): 18 /

46) Ptychomitrium polyphyllum (Sw.) B. S. G., Bryol. Eur. 3: (2-3): 82.229(1837). (= Dicranum polyphyllum Sw. in Schrad. Journ. . Bot. 4: 178 (1801)).

a) Ptychomitrium polyphyllum Br. Eur.

Etiqueta 1. - [m. Mandon]: Mandon: Mousses de (tachado): Lisbonne. [m. Willkomm]: Madere / / [m. Mandon]: Ptychomitrium polyphyllum Br. Eur. / foco da Neve. mai. $n^{\circ} 12 . /$

Etiqueta 2. - [m. Mandon]: Mandon: Mousses de Madère. // Ptychomitrium procerum Sch. / (spec. nova) / Ribera de Metade. $\mathrm{n}^{\circ} 11 /$

47) Funaria hygrometrica Hedw., Spec. Musc. 172 (1801)

Etiqueta 1. - [impreso]: E. Bourgeau, PL. CANARIENSES (ex itinere secundo) 1855. //1579. FUNARIA HYGROMETRICA Hedw. - Phyt. Can. IV, 29. / (Montagne.) / Teneriffa: Guimar, in rupibus humidis. 18 februar. I

Etiqueta 2. - [m. i. ]: Soleirol, herb. cors. // (con otro tipo de letra manuscrita ?): 5018. Funaria hygrometrica / Forêt de Perticuto. /

Etiqueta 3. - [m. i. ]: Dr. C. Baenitz, Herbarium
Europeum. / De Heldreich, Herbar. Florae Hellenicae, $N^{\circ} 100$ // Funaria hygrometrica Lin. / (sub Mnio) Schreb. / var. / Flora Attica: in herbosis in reg. infer. / m. Hymetti pr. coenobium Caesariani / Legerunt Th. de Heldreich it T. Holzmann. / D. 3 Januar / 1877. /

Etiqueta 4. - 7./ Funaria hygro- / metrica, ([m. i. ] : Bryum / [m. i. ] : Irun. Juni, 50 /

Etiqueta 5. -[m. i. ]: Bryum / Irun d. / 7./11 Junii 1850 /

Etiqueta 6. - [m. i. ]: pl. crypt. 7. / Funaria hygrometrica, Hedw. / Junn, 6/50. I

Etiqueta 7. -[m. Willkomm]: Funaria hygrometrica Hedw. / Hab. in Sierra de Yunquera loco el Caucón / ad alt. circ. 5000'. / legi die 27 April. 1845. I

Etiqueta 8. - [m. Willkomm] : Funaria hygrometrica Hedw. / Hab. in rupibus regionis alpinae / regni Valentini: in rupibus umbrosis / prientem spectantibus cacuminis Cerro / de Sta Maria montis Sierra de Chiva / in consortio Encalyptae vulgaris, ad / alt. 5500 - 6000'. / Legi d. 2 Junii 1844 . I

48) Funaria muhlenbergii Hedw. f. ex Lam. \& Cand., Fl. Franc.

2: 497(1805) (IX)

Etiqueta 1. - [m. Loscos]: Funaria / muhlenbergii / ? /

Etiqueta 2. - [m. Loscos]: Funaria / muhlenbergii /

Etiqueta 3. - [m. Loscos]: Funaria Muhlembergii / Turn. Marzo abril 1865. / ? / Herb. de Loscos /.

Etiqueta 4. - [m. Loscos]: Funaria Muhlenbergii Turn. / Frecuente alrededor de Peña / rroya en los huecos de las calzadas / de piedras junto a la Virgen de la Fuente, etc etc y al pie de los / peñascos, junto á los ? no / lejos, de la balsa de San Miguel / etc etc // febrero día 20, 1865. / [m. Willkomm]: Legit et scri? Loscos. I

49) Entosthodon attenuatus (Dicks.) Bryhn, $K$. Norsk. Vid. Selsk. Skrift. 1908(8): 25 (1908). (= Bryum attenuatum Dicks., Pl. Crypt. Brit. fasc. 4: 10 f. 8. (1801)).

a) Entosthodon templetoni Schwaegr.

Etiqueta. - [m. Mandon]: Mandon: Mousses de Maderè. // Entosthodon Templetoni Schwaegr. / Pico Las Arrieras. Juni. n 17.1 
50) Bryum schleicheri var. latifolium (Schwaegr.) Schimp., Syn. 2 (1876). (= Mnium latifolium Schwaegr., Spec. Musc. Suppl. 1(2): 138 (1816)

a) Hypnum ?

Etiqueta. - [m. Willkomm]: Hypnum? / Hab. in Sierra Nevada in pratis uliginosis turfosis / et ad rivulos regionis alpinae superioris: en el borreguil I de S. Gerónimo in parte inferiore, ad alt. 7000 8000'. / Legi d. 3 Aug. -1844. I

\section{1) Bryum capillare var. meridionale Schimp., Coroll. 75 (1856)}

a) Bryum capillare Hedw. var. meridionale

Etiqueta 1. - [m. Loscos»: 60/Bryum capillare Hedw. / v. meridionale / Hojas anchas elípticas / aserradas en el apice / Abril 1869 / Herb. de Loscos/

Etiqueta 2. -[m. Loscos]: Difiere de la v. elonga / tum por su larga cerda / flexuosa, sus hojas retorci / das cuando secas, su oper / culo mas largo. / Pl. mas tardia. Marzo /

[m. Willkomm]: Leg. Loscos. I

52) Bryum canariense Brid., Spec. Musc. 3: 29 (1817)

Etiqueta-. [impreso]: E. Bourgeau, PL. CANARIENSES (ex itinere secundo) 1855.// 1590. BRYUM CANARIENSE Brid. - Phyt. Can. IV, 32. / (Montagne.) / Teneriffa: Guimar, in convalle Chicaica, ad terram. / 18 februar. /

53) Bryum pallescens Schleich. ex Schwaegr., Spec. Musc. Suppl. 1(2): 107. 75 (1816).

Etiqueta 1. -[m. Willkomm]: Bryum pallescens Schw. / Hab. in Sierra Nevada in pratis / uliginosis partis superioris. Dehesae / de S. Gerónimo ad alt. circ. 7000-7500'. / legi d. 31 Julii 1844. /

Etiqueta 2. -[m. Willkomm]: Bryum pallescens Schw. / Crypt. hisp. exsicc. n. 45. (942). / Hab. in Sierra Nevada in rupibus / schistosis madidis in parte superiore / vallis barranco de S. Juan ad / alt. 7000-7500'. / Legi d. 3 Sept. 1844. I

Etiqueta 3. -[m. Willkomm): Bryum pallescens Schwägr. / Hab. in Sierra Nevada in pratis / uliginosis vallis barranco de Trevelez, / ad alt. circ. 7-8000'. / Legi d. 17 septemb. 1844. I

54) Bryum pseudotriquetrum (Hedw.) Gaertn., Meyer \& Scherb., Oekon: Techn. Fl. Wetteran 3(2): 102 (1802). (=Mnium pseudotriquetrum Hedw., Spec. Musc. 190 (1801) a) Mnium pseudotriquetrum var. nevadense Hamp.

Etiqueta. - [m. Willkomm]: Bryum pseudotriquetrum Schwaegr. / var. nevadense Hampe. / Crypt. hisp. exsicc. n. 33. (943). / Hab. in Sierra Nevada ad rivulos pratorum / regionis nivalis: en el borreguil de Dilas, / ad alt. 9500'. / Legi d. 3 Aug. 1844. / Vulgo: Felpilla, ut Musci plerique. I

55) Bryum bicolor Dicks., Pl. Crypt. Brit. fasc. 4 : $16(1801)$

a) Bryum atropurpureum Wahl.

Etiqueta. - [m. Mandon]. Mousses de Madére Portugal // Bryum atropurpureum Wahl. / Casilhas. fer. $n^{\circ} 22.1$

56) Plagiomnium undulatum (Hedw.)T. Kop., Ann. Bot. Fenn. 5: 146 (1968). (=Mnium undulatum Hedw., Spec. Musc. 195 (1801)).

a) Mnium undulatum Hedw.

Etiqueta 1. - [m. Loscos]: Que pl. es esta / de la serie ? / Casteliote /

Etiqueta 2. - [m. Loscos] : n. 3. / Se remitio en el primer ca/jon á WK. Abundantisimo / en el prado de Trobera de / bajo de Trafalgari. / que es ? / Loscos / Muy notable por sus hojas /

Etiqueta 3. - [m. Loscos]: $400 /$ [m. Willkomm]: Mnium undu / latum Hedw. I

57) Plagiopus oederi (Brid.)Limpr., Laubm. Deutschl. 2: 548 (1895). (=Bartramia oederi Brid., Musc. Rec. 2 (3): 135. 2f. 9 (1803)).

a) B. oederi Swartz et Sch.

Etiqueta. - [m. i. ]: D. C. Baenitz Herbarium Europaeum / N / Bartramia oederi Swartz et Sch., / c. fr. / ad rupes circa lacum M. tis Cenisii ad 2000 m c. altitudinis / 22 Julii 1876 legit J. Arcangeli.

58) Bartramia pomiformis var. elongata Turn., Ann. Bot. 1: 527 (1805)

a) Bartramia stricta $\mathrm{Sw}$.

Etiqueta. - [m. Willkomm]: Bartramia stricta Sw. / Paular de Segovia / leg. Willkomm. 1850 /

59) Bartramia ithyphylla Brid., Musc. Rec. 2 (3): 132. If. 6 (1803)

a) Bartramia

Etiqueta. - [m. Willkomm] : Bartramia / Ayamonte / Leg. Willkomm 1846 
60) Bartramia stricta Brid., Musc. Rec. 2(3): 132. 1f. 5 (1803)

a) Bartramia stricta Brid.

Etiqueta 1. - [impreso]: E. Bourgeau, PL. CANARIENSES (ex itinere secundo) 1855.// 1585. BARTRAMIA STRICTA BRID. - Phyt. Can. IV, 27. / (Montagne)/ Teneriffa: Agua Manza, in udosis 16 martii.

Etiqueta 2. - [impreso]: Soleirol, herb. Cors. / / [manuscrito]: 5024. Bartramia stricta, Brid. / Calvi et les Roullet.

61) Philonotis fontana (Hedw.) Brid., Bryol. Univ. 2: 18 (1827). (= Mnium fontanum Hedw., Spec. Musc. 195 (1801)).

a) Bartramia fontana $\mathrm{Sw}$.

Etiqueta 1. - [M. Willkomm]: Bartramia fontana Sw. / Hab. in Sierra Nevada in pratis uliginosis / regionis nivalis: en el corral de Veleta, ad / alt. 8800 - 9000' / Legi d. 26 Julii 1844 /

Etiqueta 2. - [M. Willkomm]: $\mathrm{N}^{\circ} .2 /$ Bartramia fontana Sw. / Montes prope Irun 1850 / Willkomm

Etiqueta 3. - [M. I. ] : En las cascadas / jamás fructifica / que es ? levier / [M. Willkomm]: Bartramia fontana /

Etiqueta 4. -[impreso]: Herbarium Willkomm. / [m. Willkomm]: Bartramia fontana / Aragonia australis / [impreso]: Legit [m. Willkomm]: Loscos [impreso]: 18

Etiqueta 5. -[impreso]: E. Bourgeau. Pl. Canarienses 1855 // Bartramia fontana Hedw. / (Mont.) / Teneriffa, region montagneuse supérieure / 6 Juill.

\section{b) Bartramia}

Etiqueta. - [impreso]: E. Bourgeau. - Pl. d'Espagne 1863 // [manuscrito]: Bartramia / Sierra de Tormantos región alpina / prés Plasencia. 4 Jun./

62) Orthotrichum striatum Hedw., Spec. Musc. 163 (1801)

a) Orthotrichum leiocarpum Brid.

Etiqueta 1. - [m. Loscos]: $60 /$ Orthotrichum [m. i. ] : leiocarpum / [m. Willkomm] : Brid. / [m. Loscos]: En los troncos: es muy dificil / observar el $\mathrm{n}^{\mathrm{o}}$ y disposición / de los dientes del peristoma /

Etiqueta 2. - [m. Loscos]: Ortho. (tachado): striatum ?/ ulmicola ? [m. i. ]: leiocarpum / [m. Loscos]: en las carrascas /
63) Orthotrichum anomalum Hedw., Spec. Musc $162(1801)$

a) Orthotrichum anomalum ad cupulatum

Etiqueta . - [m. Willkomm]: Aragon. / leg. Loscos. / [m. i. ]: Orthotrichum anomalum / ad cupulatum /

64) Orthotrichum cupulatum Brid., Musc. Rec. 2(2): 25 (1801)

a) Orthotrichum cupulatum Hoffm.

Etiqueta 1. - [m. Loscos]: Orthotrichum / cupulatum Hoffm. / enero 1869 / [m. i. ]: ? /

Etiqueta 2. - [m. Loscos]: Orthotrichum cupula / tum Hoffm. Marzo 1869 / Herb. de Loscos /

Etiqueta 3. - [m. Loscos]: Fruto maduro del / Orthotrichum cupulatum /

Etiqueta 4. - [m. Willkomm] : O. cupulatum Hoffm. / 13. / (tachado): O. anomalum / Hedw.

Etiqueta 5. - [impreso] : Herbarium Willkomm. / [m. Willkomm] : Orthotrichum cupulatum Hoffm. / [impreso]: Legi 18 [m. Willkomm]: $50 /$

Etiqueta 6. - [m. Loscos]: Orthotrichum cupulatum [m. i. ]: ?/

Etiqueta 7. - [m. Loscos]: Orthotrichum (tachado): anoma / lum Hedw. Marzo (sin tachar): 1869 / [m. i. ]: cupulatum / [m. Loscos]: Herb. de Loscos $/$

65) Orthotrichum rivulare Turn., Musc. Hib. 96.

8. (1804)

a) Orthotrichum rivulare $\mathrm{Sm}$.

Etiqueta 1. - [m. Willkomm]: Orthotrichum / 21. / rivulare Sm. /

Etiqueta 2. - [impreso]: Herbarium Willkomm. I [m. Willkomm]: Orthotrichum rivulare Sm. / [impreso]: Legi 18 [m. Willkomm]: 50 /

66) Orthotrichum pallens Bruch. ex Brid., Bryol. Univ. 1: 778. (1827).

a) Orthotrichum pallens Bruch

Etiqueta 1. - [m. Loscos]: Orthotrichum (tachado): affine / [m. i. ]: pallens / [m. Willkomm]: Bruch / [m. Loscos]: planta extrañísima / debe serlo. [m. i. ]: forma minor. /

Etiqueta 2. - [m. Loscos]: Orthotrichum (tachado): obtusifolio/ulmicola Lag. / [m. i. ]: pallens forma / minor / [m. Loscos] : affine, pumilum / [m. Loscos]: fin de Enero 1869 / En compañia del / Orthotrichum diaphanum /

Etiqueta 3. - [m. Loscos]: Orthotrich. affine / 
var. pumilum / mezclado con el / O. diaphanum /

67) Fontinalis antipyretica Hedw., Spec. Musc. 298 (1801)

Etiqueta 1. - [impreso]: I. Fontinalis antipyretica C. / Nel Terramaistus (Guspini), Sard. merid. Marzo./ Un. itin crypt. 1866. Dr. Marcucci./

Etiqueta 2. - $339 /$ [m. Willkomm]: Fontinalis / antipyretica L. /

Etiqueta 3. - [m. Loscos]: Sumergido en el / agua de los / arroyos / [m. Willkomm]: Aragon. / leg. Loscos. I

68) Fontinalis squamosa Hedw., Spec. Musc. 299 (1801)

Etiqueta 1. - [impreso]: II. Fontinalis squamosa L. / Nel Terramaistus (Guspini), Sard. merid. Marzo. / Un. itin. crypt. 1866. Dr. Marcucci. /

a) Fontinalis squamosa

Etiqueta 2. - [impreso]: Flora Galliae et Germaniae exsiccata. // 587. FONTINALIS SQUAMOSA, L. Sp. 1571; Wallr., Crypt. 1, / p. 205; D. 554. / Mars 1841. / Rivières rapides, aux environs de Vire; / rec. R. Lenormand. /

69) Leucodon sciuroides (Hedw.) Schwaegr., Spec. Musc. Suppl. 1(2): 1 (1816). (=Fissidens sciuroides Hedw., Spec. Musc. 161 (1801)).

a) Leskea sericea Hedw.

Etiqueta 1. - [impreso] : Herbarium Willkomm. / [m. Willkomm]: Leskea sericea Hedw. / Aragonia australis. / [impreso]: Legi [m. Willkomm]: f Loscos [impreso]: $18 /$ $|56|$

Etiqueta 2. - [m. i. ] , tachada): Leskea sericea

b) Leucodon sciuroides Schwaegr.

Etiqueta 1. - [impreso]: E. Bourgeau, PL. CANARIENSES (ex itinere secundo) 1855.// 1588. LEUCODON SCIUROIDES Schwaegr. - Phyt. Can. IV, 19. / (Montagne.) / Teneriffa: Agua Manza, in truncis castanearum. 16 martii. /

Etiqueta 2. -[m. i. ]: 56 / Leucodon sciuroides / B, morensis Schimp. /

Etiqueta 3. - [impreso]: Herbarium Willkomm. / [m. i. ]: Leucodon sciuroides / B. morensis Schimp. / Aragonia australis. / [impreso]: Legi [m. i. ]: t Loscos [impreso]: $18 /$

70) Antitrichia curtipendula (Hedw.)Brid., Musc. Recent. Suppl. 4: 136(1819). (= Neckera curtipendula Hedw., Spec. Musc. 209 (1801)).

a) Neckera curtipendula

Etiqueta 1. - [m. Willkomm]: Neckera / [m. i. ]: 329 / [m. Willkomm]: curti-pendula /

Etiqueta 2. - [impreso]: Herbarium Willkomm. / [m. Willkomm): Neckera curtipendula / Aragon. australis. / [impreso]: Legi [m. Willkomm]: t Loscos [impreso]: $18 /$

71) Pterogonium gracile (Hedw.)Sm., Engl. Bot. 16: 1085 (1802). (= Pteriginandrum gracile Hedw., Spec. Musc. 80 (1801)).

a) Pterigonum gracile Hedw.

Etiqueta. -[manuscrito]: E. Bourgeau. Pl. Canarienses 1855. // Pterigonum gracile Hedw. I (Mont.) / Teneriffa, aun Organos 16 Mars. I

72) Leptodon smithii (Hedw.)Web. \& Mohr, Ind. Mus. Pl. Crypt. 2 (1803). (= Hypnum smithii Hedw., Spec. Musc. 264. 68 f. 5-7 (1801)

a) Leptodon smithii Mohr.

Etiqueta 1. -[m. Loscos]: 44 / Gen. desconocido $/$

Etiqueta 2. - [m. i. ]: Fabronia / pusilla / [m. i. ]: Leptodon Smithii [m. Willkomm]: Mohr. /

Etiqueta 3. - [impreso]: Herbarium Willkomm. / [m. Willkomm] : Leptodon Smithii Mohr. / Aragonia aragonensis / [impreso]: Legi [m. Willkomm]: t Loscos [impreso]: 18 /

b) Pterogonium Schmitii Hedw.

Etiqueta. - [impreso]: soleirol, herb. cors. // [manuscrito]: 5033. Pterogonium Schm/itii, Hedw. / Foret de Perticote /

73) Neckera crispa Hedw., Spec. Musc. 206(1801) a) Neckera crispa Hedw.

Etiqueta . - [manuscrito]: E. Bourgeau. Pl. Canarienses 1855 // Neckera crispa Hedw. / (Mont.) / Teneriffa, font de las Mercedès. / 10 Février. /

b) Neckera intermedia Brid.

Etiqueta . - [m. Mandon]: Mandon: Mousses de Madère // Neckera intermedia Brid. / Ribero Frio. Junio. no $30 /$

74) Hookeria lucens (Hedw.)Sm., Trans. Linn. Soc. London 9: 275 (1808). (= Hypnum lucens Hedw., Spec. Musc. 243 (1801)).

a) Pterygophyllum lucens Brid.

Etiqueta . -[m. Mandon]: Mandon: Mousses de Madère. // Pterygophyllum lucens Brid. / n 35 / 
75) Fabronia pusilla Raddi, Atti Acc. Sc. Siena 9: 231. 1. (1808)

Etiqueta 1. - [impreso]: Herbarium Willkomm. / [m. Willkomm]: Fabronia pusilla Raddi / Aragonia australis . / [impreso]: Legi [m. Willkomm]: / Loscos [impreso]: $18 /$

Etiqueta 2. - (letra manuscrita ?): Peñarroya / Fabronia / pusilla / Raddi / ?! ? /

76) Habrodon perpusillus (De Not.)Lindb., Oefv. K. Vet. Ak. Foerh. 20: 401(1863). (= Pterogonium perpusillum De Not., Musc. Ital. Spec. 12 (1837)).

a) Habrodon notarisci Schimp.

Etiqueta - - [m. i. ]: Dr. C. Baenitz - Herbarium Europaeum $N^{\circ}$ // Habrodon Notarisci Schimp. / Pterogonium perpusillum D. Not. / Etnoria ad truncos Abietis pectinatae decliv. merid. montis / Senario» prope Florentiam / Maii 1877 legit E. Levier /

77) Thamnobryum alopecurum (Hedw.)Nieuwl., Am. Midland Natural. 5: 50(1917). (=Hypnum alopecurum Hedw., Spec. Musc. 267 (1801)).

a) Hypnum alopecurum $L$.

Etiqueta 1 . - [manuscrito]: E. Bourgeau. Pl. Canarienses 1855. // Hypnum alopecurum L. / (Mont.) / Teneriffa, foret del Agua Garcia / 4 mars./ Etiqueta 2. -[m. i. ]: Soleiro, herb. cors. // 5034. Hypnum alopecurum / Foret de Perticoto. /

Etiqueta 3. - [m. Loscos]: $337 /[\mathrm{m}$ Willkomm]: Hypnum / alopecurum L. /

Etiqueta 4. - [impreso]: Herbarium Willkomm. / [m. Willkomm]: Hypnum alopecurum / Aragonia australis / [impreso]: Legi [m. Willkomm]: t Loscos [impreso]: 18

78) Thuidium tamariscinum (Hedw.)B. S. G., Bryol. Eur. 5: (49- 51): 163. 482. 483(1852). (=Hypnum tamariscinum Hedw., Spec. Musc. 261. 67 f. 1-5 (1801)).

a) Hypnum tamariscinum $L$.

Etiqueta 1. - [impreso]: Herbarium Willkomm. / [m. Willkomm] : Hypnum tamariscinum L. / Aragonia australis / [impreso] : Legi [m. Willkomm] : t Loscos (letra impresa): 18 /

Etiqueta 2. - [m. Loscos]: N. L. / Se remitó a WK. en el / primer cajón; abunda en / las alturas; figurar deben en / las Series, y debe respetarse / como una especie Assoana; /Barrancos hondos del
Bojar / Que es? Loscos /

79) Cratoneuron commutatum (Hedw.)G. Roth, Hedwigia 38 Beibl. 1: 6(1899). (=Hypnum commutatum Hedw., Spec. Musc. 284 (1801)).

a) Hypnum commutatum Hedw.

Etiqueta 1. - [impreso]: Herbarium Willkomm. / [m. Willkomm]: Hypnum commutatum Hedw. / Aragonia australis / [impreso] : Legi [m. Willkomm]: t Loscos [impreso]: 18

Etiqueta 2. - [m. Loscos]) : $336 /[\mathrm{m}$. Willkomm]: Hypnum commutatum / hedw. /

b) Hypnum commutatum Hedw. var. prolixum Hampe

Etiqueta. - [m. Willkomm]): Hypnum commutatum Hedw. / var. prolixum Hampe. / Crypt. hisp. exsicc. n. 55. (944). / Hab. in Sierra Nevada in rivis regionis alpinae: / in rivo lateris australis cacuminis et Dornajo copiose / Alt. 5500-6000'. / Legi d. 29 Julii 1844.

80) Cratoneuron commutatum var. falcatum (Brid.)Mönk., Hedwigia 50: 269(1911). (= Hypnum falcatum Brid., Musc. Rec. 2(3): 63. $1 \mathrm{f}$. $6(1801))$.

a) Hypnum commutatum Hedw. var. falcatum Hampe

Etiqueta - - [m. Willkomm ]: Hypnum commutatum Hedw. / var. falcatum Hampe. / Crypt. hisp. exsicc. n. 39. (945). / Hab. in Sierra Nevada in rivulis regionis alpinae superioris: / en el borreguil de S. Gerónimo in parte inferiore abundantes, / al alt. $7000-7500$ '. / Legi d. 3 aug. 1844. /

b) Hypnum falcatum Brid.

Etiqueta . - [impreso]: IV. Hypnum falcatum Brid. steril. / Tacquitara (Barbargia), bagnato dalle acque calcarifere, Maggio. / Un. itin. crypt. 1866. Dr. Marcucci. /

81) Calliergonella cuspidata (Hedw.)LoesKe, Hedwigia 50: 248 (1911). (= Hypnum cuspidatum Hedw., Spec. Musc. 254 (1801)).

Etiqueta . - [m. Loscos ]: $330 /$ [m. Willkomm]: Leg. Loscos /

82) Isothecium myosuroides Brid., Bryol. Univ. 2: 369 (1827)

a) Brachythecium megapolitanum Bland.

Etiqueta . - [m. Loscos]: Brachythecium / megapolitanum / [m. Willkomm]: (Bland.) / [m. 
Loscos]: En los corvos con / desconocidos / H. subtile? / (letra manuscrita de Willkomm): Leg. Loscos $/$

83) Homalothecium sericeum (Hedw.)B. S. G., Bryol. Eur. 5: (46-47): 93.456(1851). (= Leskea sericea Hedw., Spec. Musc. 228 (1801)).

a) Homalothecium sericeum Sch. var. meridionale Sch.

Etiqueta 1. - [manuscrito]: Mandon: Mousses de Madère. // Homalothecium sericeum Sch. / var. meridionale Sch. / de lapa do Passo pao ? $\mathrm{n}^{\circ} 37$. I

b) Hypnum

Etiqueta 2. - [impreso] : FLORA GRAECA EXSICCATA //N ${ }^{\circ}$ [m. i. ] : 138, Hypnum / [impreso] : Habit. [m. i. ] : in axrocarintho (rara) / [impreso] : Fl. [m. i. ] : Mart-apr. alt 1880' / [impreso] : Legit [m. i. ] : 18 April_-1852 / [impreso]. THEODORUS G. ORPHANIDES. I

c) Leskea sericea Hedw.

Etiqueta 3. -[impreso] : E. Bourgeau, PL. CANARIENSES (ex itinere secundo) 1855.// 1584. LESKEA SERICEA Hedw. - Phyt. Can. IV, 13. I (Montagne.) / Teneriffa: Agua Manza, ad truncos. 16 martii. I

84) Homalothecium aureum (Spruce)Robins. Bryologist 65: 96 (1962). (= Isothecium aureum Spruc., Musci Pyren. n. 85 (1847)).

a) Camptothecium aureum Schimp.

Etiqueta - - [impreso]: F. Schultz, herbarium normale. Cent. 8. // 786, CAMPTOTHECIUM AUREUM (Hypnum Lagasca ann. / de client. nat. n. 14), P. Schimper Syn. musc. / europ. 529. / 23 février 1862 et 7 décembre 1863. / dans les bruyères sur le grès bigarré, à Cannet-du-Luc (Var). / Rec. Robert et Hanry. I

85) Homalothecium lutescens (Hedw.)Robins., Bryologist 65: 98 (1962). (= Hypnum lutescens Hedw., Spec. Musc. 274 (1801)).

a) Hypnum lutescens Hedw.

Etiqueta . - [m. Willkomm] : Hypnum lutescens Hedw. / Legit Loscos in Aragonia australis /

86) Brachythecium rutabulum (Hedw.) B. S. G., Bryol. Eur. 6: (52-54): 15(1853). (= Hypnum rutabulum Hedw., Spec. Musc. 276 (1801)).

Etiqueta . - [m. Willkomm]: Brachythecium rutabulum / [m. Willkomm]: Aragonia australis /
Leg. Loscos. 1

87) Brachythecium velutinum (Hedw.)B. S. G., Bryol. Eur. 6: (52-54): 9(1853). (= Hypnum velutinum Hedw., Spec. Musc. 272 (1801)).

Etiqueta . - [m. Willkomm]: Brachythecium velutinum (L.) / Br. E. / Aragonia australis / Leg. $\operatorname{Los} \cos /$

88) Eurhynchium praelongum var. stokesii (Turn.)Podp., Consp. Musc. Eur. 629(1954). Hypnum stokesii Turn., Musc. Hibern. Spic. 159. 15 f. 2)). (1804)

a) Hypnum stokesii Turn.

Etiqueta: [impreso]: E. Bourgeau. PL. CANARIENSES (ex itinere secundo) 1855.//1580. HYPNUM STOKESII Turn. / Eurhynchyum Stokesii Schimper. / (montagne.) / Teneriffa: Agua-Garcia, in opacis lauretorum. 4. martii. I

89) Hypnum cupressiforme Hedw., Spec. Musc. 291 (1801)

a)Hypnum cupressiforme

Etiqueta 1.- [impreso] : Herbarium Willkomm. / [m. Willkomm] : Hypnum cupressiforme / Aragonia australis / [impreso] : Legi [m. Willkomm] : / Loscos [impreso]: 18 /

Etiqueta 2. - [impreso]: E. Bourgeau , PL. CANARIENSES (ex itinere secundo) 1855.// 1591. HYPNUM CUPRESSIFORME L. - Phyt. Can. IV, 6. / (Montagne.) / Teneriffa: selva del Agua Garcia, ad truncos. 4 martii. /

90) Ptilium cristacastrensis (Hedw.)De Not., Cronac. Briol. Ital. 2: 17(1867). (=Hypnum crista_castrensis Hedw., Spec. Musc. $287.76 \mathrm{f}$. 1-4(1801)).

a) Hypnum crista castrensis L.

Etiqueta 1. - [m. Loscos]: $331 /$ [m. Willkomm]: Hypnum crist / castrensis L. /

Etiqueta 2. - [impreso] : Herbarium Willkomm. / [m. Willkomm] : Hypnum Crista castrensis L. / Aragonia australis. / [impreso]: Legi [m. Willkomm] : t Loscos [impreso] : 18 /

\section{BIBLIOGRAFÍA}

BRUCH, PH., W. PH. SCHIMPER \& TH. GUMBEL - 1836/51- Bryologia Europaea. 6 Vols. Stuttgart 
CASARES GIL, A. -1932- Flora Ibérica. Briófitas ( $2^{\mathbf{a}}$ Parte). Musgos. Mus. Nac. Cien. Nat. 1-434 Madrid

CASAS, C. -1981- The Mosses of Spain: an annotated check-list. Treballs Inst. Bot. Barcelona 7: 1-57

CORLEY, M. F. V., A. C. CRUNDWELL, R. DÜLL M.O. HILL \& A.J.E. SMITH -1981- Mosses of Europe and the Azores; an annotated list of species, with synonyms from the recent literature. Journ. Bryol. 11: 609-689

CRUM, H. A. \& L. E. ANDERSON-1981- Mosses of Eastern North America 2 Vols. Columbia. University Press. New York

DEMARET, F. \& E. CASTAGNE -1968- Flore Genérale de Belgique. Bryophytes. Vol. II. Ministère de l'Agriculture. Brussels.

DÜLL, R. -1980- Die Moose (Bryophyta) des Rheinlandes Nordrhein. Westfalen, Bundesrepublik Deutschland. Decheniana Beih. 24: $1-365$

HUSNOT, T. -1884/90- Muscologia Gallica. Paris

KUNZE, G. -1846- Chloris Austro-Hispanica. Ratisbonae

LIMPRICHT, G. -1895- Die Laubmoose. Deutschlands, Osterreichs und Schweiz. Leipzig

MONKEMEYER, W. -1927- Die Laubmoose Europas (Andraeales-Bryales). In Rabenhorst, Kryptogamen-Flora von Deutschland, Osterreichs und Schweiz. Leipzig

PARIS, E. G. - 1904- Index bryologicus Enumeratio Muscorum. (ed. $2^{\mathrm{a}}$ ) 6 Vols.

SMITH, A. J. E. -1980- The Mosses of Britain and Ireland. Cambridge University Press Cambridge.

VIERA, M. C. y J. REINOSO. -1993- Los briófitos del herbario de Willkomm (COI). I. Hepaticae. Acta Bot. Malacitana, 18: 65-71.

WIJK, R., W. D. MARGADANT \& P. A. FLORSCHUTZ. - 1959-69- Index Muscorum, 5 Vols. Internat. Bur. Pl. Taxonomy. Utrecht
WILLKOMM, M. -1844- Botanische Berichte aus Spanien. Bot. Zeit. 2(42): 721-725

WILLKOMM, M. -1851- Vegetationsskizzen aus Spanien. Bot. Zeit. 9(3): 33-44

WILLKOMM, M. -1852- Sertum Florae Hispanicae. Leipzig

Aceptado para su publicación en Julio de 1992

Dirección de los autores. Departamento de Biología Vegetal (Botánica). Facultad de Biología. Universidad de Santiago de Compostela. España. 